\title{
Programa Academia da Cidade do Recife: análise da contratransferência de política
}

\author{
Recife City Academy Program: analysis of the policy countertransference
}

\section{AUTORES \\ Érica Maria do Nascimento Melo ${ }^{1}$ (D) Flávio Renato Barros da Guarda ${ }^{2}$ (D) \\ Francisco de Assis da Silva Santos ${ }^{3}$ (D) \\ Wallacy Milton do Nascimento Feitosa ${ }^{4}$ (D) \\ 1 Universidade Federal de Pernambuco, \\ Departamento de Educação Física, Recife, \\ Pernambuco, Brasil. \\ 2 Universidade Federal de Pernambuco, \\ Departamento de Educação Física, Vitória de Santo Antão, Pernambuco, Brasil. \\ 3 Universidade Federal de Pernambuco, Centro Acadêmico do Agreste, Caruaru, Pernambuco, Brasil. \\ 4 Colégio de Militar do Recife, Recife, Pernambuco, Brasi.}

\section{CONTATO}

Érica Maria do Nascimento Melo ericanascimento1991@hotmail.com Rua Madre de Deus, 185, Jaboatão, Pernambuco, Brasil.

CEP: 54090-060.

DOI

$10.12820 /$ rbafs. $25 \mathrm{e} 0174$

\section{(cc) BY}

Este trabalho está licenciado com uma Licença Creative Commons - Atribuição 4.0 Internacional.

\begin{abstract}
RESUMO
Este estudo tem o objetivo de analisar o processo de contratransferência de política envolvendo o Programa Academia da Cidade do Recife. Estudo com abordagem qualitativa. Foi realizada análise documental a partir de relatórios, avaliações, Portarias e Resoluções, Notas Técnicas, Diários Oficiais do Município, Estado e da União, como também os Planos Municipais de Saúde do município de Recife. Para a definição da quantidade final dos participantes da pesquisa foi utilizado o critério de amostra por saturação. Todos os participantes concordaram e contribuíram na pesquisa. Não houveram desistências durante o processo da pesquisa. Participaram da pesquisa seis importantes sujeitos-chave, dentre eles, gestores, coordenadores, formuladores e profissionais envolvidos no Programa Academia da Cidade do Recife e em programas congêneres. Os dados foram analisados com base no modelo de condensação de significados. A contratransferência é identificada posteriormente ao processo de transferência e está relacionada a uma retroalimentação que pode ser percebida a partir da identificação do recebimento de recursos para além do âmbito financeiro. Foi identificada a contratransferência de política envolvendo o PAC e o Programa Estadual Academias das Cidades do Governo do Estado de Pernambuco e o Programa Federal Academia da Saúde. Podemos considerar que o PAC Recife, devido a sua formulação e implantação exitosa, foi alvo do processo de transferência e de contratransferência de política.
\end{abstract}

Palavras-chave: Programas de saúde; Atividade física; Políticas públicas de saúde; Pesquisa qualitativa. ABSTRACT

To analyze the policy countertransference process involving the Academia da Cidade do Recife Program. Study with a qualitative approach. Documentary analysis was performed from reports, evaluations, Ordinances and Resolutions, Technical Notes, Official Diaries of the Municipality, State and Union, as well as the Municipal Health Plans of the municipality of Recife. For the definition of the final quantity of the research participants, the criterion of sample by saturation was used. Six important key subjects participated in the research, among them, managers, coordinators, formulators and professionals involved in the Academia da Cidade Program in Recife and similar programs. The data were analyzed based on the condensation of meanings model. The countertransference is identified after the transfer process and is related to feedback, which can be perceived from the identification of the receipt of funds beyond the financial scope. The policy countertransference involving the PAC and the State Academies of Cities Program of the Government of the State of Pernambuco and the Federal Health Academy Program were identified. PAC Recife, due to its successful formulation and implementation, was the target of the policy transfer and countertransference process.

Keywords: Health programs; Physical activity; Public health policies; Qualitative research.

\section{Introdução}

Nos últimos anos, diante de um cenário de inatividade física e da possibilidade da utilização da promoção da atividade física como estratégia de política pública de saúde para viabilizar o acesso à prática de atividade física, surgiram ações e programas comunitários de atividade física no país ${ }^{1-5}$.

Nesse contexto, no Recife, capital de Pernambuco, surge o Programa Academia da Cidade (PAC), que foi institucionalizado em 2003 e, no ano de 2006, tornou-se uma política de saúde do município visando possibilitar a prática de atividades físicas e a adoção de hábitos alimentares saudáveis para a população, requalificando praças e parques para construção dos polos onde ocorrem as ações do programa ${ }^{6}$.

A partir do seu surgimento e consolidação, outros programas começaram a ser formulados com nomes ou moldes similares, baseados no programa do Recife ${ }^{7,8}$. De acordo com Dolowitz \& Marsh $^{9,10}$, esse processo é descrito como transferência de política e ocorre devido às contribuições que o uso de informações de políticas já existentes poderia trazer para quem pretende adotar uma política. $\mathrm{O}$ uso de informações políticas já existentes tenderia a diminuir as possibilidades de fracasso e a 
orientar todo o ciclo, desde a formulação até a implantação de uma política.

De acordo com Evans ${ }^{11}$, a prova de que houve uma transferência de política está em sua implantação. Com a implantação de uma política a partir de uma já existente é possível analisar e identificar conteúdo de uma transferência. A partir da análise do processo de transferência é possível identificar a importação de ideias de outra política, desde provas documentais até a análise das interações entre os agentes sociais envolvidos. Fica evidenciado, nesse caso, que a formulação de políticas públicas foi influenciada por iniciativas e experiências de êxito, como o PAC.

Com base na existência do processo de transferência de política, o presente estudo propõe analisar o PAC a partir de um novo modelo de análise de política pública: o processo de contratransferência identificado e descrito inicialmente por Feitosa ${ }^{8}$. De acordo com o autor, a Contratransferência ocorre quando um programa que influenciou e serviu de modelo para a criação de outros programas, posteriormente, passa a ser beneficiado. Neste caso, o doador de uma política passa a ser retroalimentado pela política que o originou. Para que ocorra o processo de contratransferência é necessário que a política tenha sido objeto de transferência de política. Com base na análise do processo de contratransferência, é possível caracterizá-lo e compreendê-lo. $\mathrm{Na}$ literatura ainda não existem dados sobre a análise desse processo nos estudos sobre política.

\section{Métodos}

O presente estudo se caracteriza como um estudo de caso, exploratório e analítico. Para a coleta dos dados foi utilizado o método de triangulação, que possibilita a utilização de várias fontes de informações e técnicas de coleta de dados ${ }^{12}$. O estudo foi realizado no município do Recife, capital de Pernambuco. As entrevistas foram previamente agendadas e realizadas no período de julho a novembro de 2018. Um total de seis sujeitos-chave participaram da pesquisa. Os sujeitos-chave foram definidos por serem peças fundamentais e singulares, conhecendo de perto e com experiência na história e gestão dos programas envolvidos nessa pesquisa, são eles gestores, coordenadores, formuladores e profissionais envolvidos no Programa Academia da Cidade do Recife e de programas similares que tiveram como base o PAC para sua construção e formação. Para o estudo foi utilizado o critério de amostra por saturação, não sendo necessário outros informantes chaves além dos selecionados, por encontrar as respostas pertinentes as pesquisas de forma satisfatória. $\mathrm{O}$ roteiro utilizado foi construído com base nos estudos e levantamento de dados sobre os programas e foi idêntico para todos os envolvidos nas pesquisas. Foi realizado um pré teste com um dos entrevistados no início da pesquisa para identificar se as questões eram de claro entendimento, foi verificado que o questionário era claro e atendia ao proposto não sendo necessário novos ajustes.

$\mathrm{Na}$ etapa seguinte, após a coleta dos dados e transcrição do áudio, foi realizada a análise de conteúdo. Para a análise do conteúdo das entrevistas foi utilizado como referência os modelos de análise de conteúdos de Kva$1 \mathrm{e}^{13}$. Dentre os tipos propostos por este autor, utilizou-se a Condensação de Significados por ser considerado o mais adequado aos objetivos do estudo: tem o objetivo de identificar pontos em comum da percepção dos sujeitos entrevistados. Nesse tipo de análise, as respostas das entrevistas foram relacionadas a questão específica do estudo relacionada a ela, cotadas e condensadas num quadro constituído pelas unidades naturais dos significados, que são as respostas dadas pelos sujeitos. $\mathrm{Na}$ coluna da esquerda foram postos os temas centrais relacionados a estes, que são categorias conceituais. $\mathrm{Na}$ coluna da direita, a descrição essencial da questão relacionada ao estudo, conforme demonstrado no Quadro 1:

Quadro 1 - Quadro de análise da condensação dos significados

\begin{tabular}{|l|l|}
\hline 1. Pergunta \\
\hline Unidade Natural & Tema Central \\
\hline Resposta do entrevistado & Qual tema central da resposta \\
\hline Descrição Essencial da Questão de Pesquisa \\
\hline Descrição da resposta \\
\hline
\end{tabular}

Fonte: Baseado no quadro de Giorgi apud Kvale ${ }^{13}$.

Os dados foram coletados a partir de relatórios, avaliações, Portarias e Resoluções, Notas Técnicas, Diários Oficiais do Município, Estado e da União, como também os Planos Municipais de Saúde do município de Recife, acessados fisicamente e por meio digital nos sites dos municípios envolvidos. Alguns documentos são de domínio público, e outros foram disponibilizados após solicitado pelos pesquisadores mediante a apresentação da carta de anuência.

Esta pesquisa atende à Resolução 466/13 do Conselho Nacional de Saúde/Comissão Nacional de Ética em Pesquisa, submetida ao Comitê de ética do Centro de Ciências da Saúde (CEP- CCS) da Universidade 
Federal de Pernambuco (UFPE), aprovada sob parecer consubstanciado No 2.489.658/2018.

\section{Resultados}

No município de Recife foi identificado que, após o surgimento do PAC, outros programas começaram a ser formulados com nomes ou moldes similares ao programa. A partir da análise da fala de alguns atores envolvidos no processo, é possível identificar diversas evidências da ocorrência da transferência de política. $\mathrm{O}$ conhecimento do programa por entes interessados em conhecer e implantar seu modelo em outros contextos foi relatado na fala a seguir:

Recebemos muita gente e não só do Brasil, teve uma equipe de San Diego que queria implantar lá e eles vieram para conhecer, passaram dias e dias com a gente acompanhando o professor do polo para implantar lá o Academia da Cidade, lá em San Diego... Nós conseguimos sim fazer essa transferência de política, como que a gente fazia e como a gente conseguia compartilhar com os outros, e eles levavam esse conhecimento ${ }^{14}$.

Após ter sido objeto de transferência de política, o PAC passa a apresentar um fenômeno interessante denominado "Contratransferência" 8 . De acordo com Feitosa ${ }^{8}$, a contratransferência ocorre quando um programa serve de modelo e influência na criação de outros programas e, posteriormente, passa a ser beneficiado por estes (Quadro 2). A partir das análises desse estudo, identifica-se que o PAC começa a receber alguns retornos advindos de programas e políticas que teriam surgido a partir do seu modelo, ou seja, o objeto de transferência passa agora a ser retroalimentado, recebendo influências dos programas advindos dele (Tabela 1).

Quadro 2 - Conceito e característica da transferência e contratransferência

\begin{tabular}{|ll|}
\hline \multicolumn{1}{|c|}{ Transferência de política } & \multicolumn{1}{c|}{ Contratransferência } \\
\hline Conhecimento a respeito de & Ocorre quando determinada \\
determinada política pública é & política, que foi objeto de \\
utilizado como modelo para o & transferência para a criação de \\
desenvolvimento de outra política & uma nova política, passa a ser \\
pública. & retroalimentada por essa nova \\
& política. \\
\hline
\end{tabular}

Fonte: Dolowitz \& Marsh $^{9}$; Feitosa ${ }^{8}$.

A contratransferência pode ser categorizada como positiva, quando existe um retorno positivo, visando
Tabela 1 - Esquema de transferência e contratransferência ocorrido com o PAC Recife, Pernambuco

\begin{tabular}{lll}
\hline & Transferência \\
\hline PAC RECIFE & & PACID \\
\hline & & PAS \\
\hline PAC RECIFE & Contratransferência & PACID \\
\hline
\end{tabular}

PAC $=$ Programa Academia da Cidade PACID = Programa Acade mia das Cidades; PAS = Programa Academia da Saúde.

contribuir e fomentar benefícios para a política que foi doadora, que agora está sendo retroalimentada, e em contratransferência negativa, quando existe um retorno, porém sem qualquer contribuição para a política ou para fatores burocráticos, por exemplo, que podem dificultar ou enfraquecer uma política. Nesse estudo, podemos identificar alguns fatores positivos e negativos resultantes do processo de contratransferência ocorridos no PAC, como demonstrado no Quadro 3.

Quadro 3-Fatores positivos e negativos resultantes do processo de contratransferência no Pac Recife

\begin{tabular}{|l|l|}
\hline \multicolumn{2}{|c|}{ Contratransferência no PAC } \\
\hline Positivas & Negativas \\
\hline Convênios & Maior burocracia \\
\hline Custeios & Normatizações \\
\hline Formação profissional & \\
\hline Gestão organizacional & \\
\hline Visibilidade & \\
\hline
\end{tabular}

\section{Discussão}

Para Rose ${ }^{15}$, a partir desse conhecimento e análise de ações ou políticas de outros governos e lugares, é possível o aprendizado de lições importantes para os formuladores de política, que podem ser positivas ou negativas, podendo ser transferidas quando constatadas como eficazes. Também é identificado, a partir dessa fala, um interesse na transnacionalização da política que, segundo Stone ${ }^{16}$, é quando uma política ultrapassa as fronteiras nacionais neste processo. Neste caso, a atividade de transferência transcende tanto o cenário nacional como também o internacional. Cabe destacar que o PAC foi objeto de algumas pesquisas e avaliações, inclusive internacionais, divulgando seus resultados e ações e fomentando a transferência dessa política para esses contextos ${ }^{17-19}$.

A literatura apresenta alguns estudos que analisam o que seria essa transnacionalização de políticas. No 
estudo sobre a implementação de políticas públicas nacionais de desenvolvimento rural na América Latina, os princípios fundamentais dessa política foram emulados de um programa desenvolvido na União Europeia analisando a sua viabilidade em contextos diferentes, que podem influenciar ou desencadear sucessos ou fracassos na transferência de política ${ }^{20}$. Em outro exemplo de estudo, porém, no contexto de políticas ambientais, Fontoura \& Guedes ${ }^{21}$ tratou de como o protocolo de Cartagena (Colômbia) influenciou a formulação e a implementação de uma política brasileira de biossegurança, analisando a transferência de política em contextos distintos, e identificando que, neste caso, houve a transferência de política.

No contexto dos programas de atividade física que se basearam nos princípios do PAC para a sua criação, citamos o Programa Academia das Cidades (PACID), do Governo do Estado de Pernambuco ${ }^{22}$. Cabe evidenciar que o Secretário das Cidades, responsável pela criação do PACID, havia sido Secretário da Saúde do município de Recife na época em que foi criado o PAC Recife, podendo-se, assim, evidenciar uma forte influência do programa do Recife na criação do PACID ${ }^{8}$. Fica evidenciado a importância do papel do gestor e do alinhamento político verificado, que cooperou e foi fundamental para essas implantações.

Nesse contexto, quando da sua implantação, o PAC Recife teve todo o seu financiamento demandado exclusivamente do município de Recife, tanto para a construção de polos como para o custeio mensal ${ }^{6}$. A partir do surgimento do PACID, foi estabelecido um convênio com o PAC para a construção de novos polos. Desta forma, observa-se que o PACID, que se baseou no PAC, agora passa a retroalimentar a política que inspirou a sua criação, a partir de parcerias para a construção de novos polos do Programa Academia da Cidade do Recife. Essa retroalimentação foi identificada pela parceria estabelecida entre os programas. De acordo com os achados desse estudo, esse é um primeiro indício do processo de contratransferência de política.

Nessa parceria, a Secretaria das Cidades, em convênio com a Prefeitura do Recife, financiava a construção de novos polos, conforme relato: "com lançamento em 2007 do Programa Academia das Cidades, a partir de 2008 o programa de Recife já conseguiu fazer parceria e implantou mais 21 novos polos" ${ }^{\text {" }}$.

Além disso existe outras influências como o fato do PACID possuir um sistema informatizado de acompanhamento do seu programa, disponibiliza e compar- tilha seus modelos de planilhas de acompanhamento com o PAC, que é utilizado por este no seu monitoramento anual, conforme exemplo nos relatos abaixo:

O Academia das Cidades por exemplo, o sistema de informação da gente todo ele foi feito pela gente, mas teve um pessoal do estado e lá tem uma pessoa, um nodal que faz isso e a gente aqui não tem ${ }^{23}$.

O estado que tem monitoramento próprio, e quando a gente monitora a gente consegue avaliar e dá um retorno para o programa a gente sabe o quanto que o Recife já avançou nesse processo de monitoramento a partir de experiências do Estado não é eu acho que é isso é transferir valor não necessariamente valor financeiro ${ }^{14}$.

A prática de uma retroalimentação pode ser reconhecida como exemplo na fala do Entrevistado 2:

A gente trabalhou com a planilha de monitoramento cedida... olha já vem o estado de novo, está vendo, retroalimentando o programa, eles cederam para a gente a planilha de monitoramento, não é de planejamento, é de monitoramento que ela é uma visão do ano todo com as atividades e o status ${ }^{23}$.

Além do convênio e das contribuições técnicas citadas, por ser um programa de atividade física, e com alguns princípios parecidos com o PAC, o PACID também contribui com o PAC nos aspectos metodológicos, trazendo uma maior diversificação na oferta de outras atividades com aspectos culturais, e de acordo com o contexto de cada localidade, o que enriquece a produtividade dos polos, características próprias do programa estadual $^{22}$.

Nesse estudo, cabe ressaltar que o PAC Recife foi fundamental ao formular um programa de promoção da atividade física e saúde numa perspectiva mais ampliada, e, como um equipamento de saúde do município, o que contribuiu para a visibilidade do programa, que, inclusive, foi reconhecido em âmbito nacional pelo Ministério da Saúde como uma proposta exitosa de atividade física de base populacional ${ }^{7}$, despertando o interesse e motivando outras iniciativas a seguir o modelo, de maneira geral os discurssos monstram uma grande influência do Programa de Recife:

O Academia da Cidade influenciou o programa 
Academia da Saúde, influenciou a sua gestão e sua execução. Nós, graças a Deus, somos privilegiados por que nós fazemos um trabalho diferenciado aqui na cidade do Recife, coisa que se o Academia da Saúde pudesse fazer em todos os estados do Brasil seria ótimo. Um exemplo é trabalhar em rede, nós trabalhamos com rede, a rede de saúde, está inserido, é um equipamento de saúde pública o $\mathrm{PAC}^{24}$.

Um fato interessante identificado nesse estudo, que comprova o sucesso e a visibilidade desse programa como uma referência importante, foi o fato de o programa servir de referência para a rede de saúde do Recife, inclusive particular, conforme relato abaixo de um usuário do programa que recebeu encaminhamento do médico para o Academia da Cidade do Recife:

O médico de um plano de saúde particular encaminhando, vá para o Academia da Cidade fazer fortalecimento dos músculos paravertebrais, usando a linguagem da gente a coisa mais linda do mundo, então assim prova que assim a muito tempo já a gente ta lutando por essa referência e contrarreferência ${ }^{23}$.

Outro programa de abrangência nacional, em que foi evidenciado o processo de transferência de política em relação ao PAC, foi o Programa Academia da Saúde (PAS). O PAS é uma estratégia de promoção da saúde da atenção básica, que se articula com outros programas e ações da saúde. Cabe destacar que o programa no Recife é um dos que mais se aproxima das grandes diretrizes e objetivos do PAS, pois o PAC Recife trabalha numa perspectiva ampliada. É interessante analisar a importância dos gestores nesse processo e do alinhamento entre eles, tanto o Ministro da Saúde como os técnicos responsáveis pela área de atividade física no Ministério da Saúde, teve forte envolvimento nos primórdios com a formulação gestão e implantação do programa de Recife. É fundamental essa compreensão e o entendimento que essa ligação foi fundamental e contribuiu para o processo de contratransferência. Após a formulação e implantação do PAS, a partir de modelos de programas exitosos pelo Brasil, é lançado um edital para custeio mensal e construção de polos, passando a retroalimentar o programa do Recife ${ }^{25,26}$.

Inicialmente, o PAC era custeado exclusivamente pelo município do Recife. Após a criação do PAS e a participação no edital de custeio, os polos do PAC, classificados como similares e que atendiam aos critérios do PAS, passaram a receber um custeio de 3 mil reais mensais do Governo Federal para a sua manutenção ${ }^{27}$. A partir dessa evidência, fica claro o início do processo de contratransferência de política entre o PAC e o PAS.

Atualmente, a maioria dos polos do PAC Recife possui convênio com o PAS; apenas quatro polos não são conveniados com este, pois não atendem aos critérios, como informado na fala do Entrevistado 3: "Dos 42 polos, hoje 4 não são porque não atende as exigências e os critérios do ministério. A gente tem alguns polos que não entram dentro das características de Polo similar para receber o recurso e ser caracterizado" ${ }^{24}$. Neste caso, além de recursos financeiros, a contratransferência traz também influências positivas para o PAC. Em relação às capacitações profissionais, o PAS elabora cursos à distância em parceria com algumas universidades, e realiza seminários durante o ano. Esta relação colabora e valoriza tanto o programa como os profissionais do Recife, como exemplo:

Promovem seminário que promove algum movimento de estar repensando a prática, ele está valorizando o que os profissionais estão fazendo, valorizando o que a gestão está fazendo, valorizando o processo de trabalho, de alguma maneira isso agrega um pouco de aspectos positivos ${ }^{14}$.

Existe uma relação muito boa entre ambos os programas. O PAS realiza algumas amostras nacionalmente e convida o PAC para participar e compartilhar suas intervenções, devido aos resultados exitosos. As formações geralmente estão relacionadas ao sistema para monitoramento do programa, prestação de contas e documentos, que devem ser direcionados ao Ministério durante o ano. Cabe mencionar que o PAC, desde a sua implantação, possui sua própria linha de formação e continua realizando suas próprias formações, mesmo nos polos conveniados com o PAS.

Neste caso, as influências advindas agregam e contribuem no seu funcionamento. Após o convênio com o PAS, o PAC passou a entrar na agenda das discussões interministeriais e no sistema de gestão de informação da atenção básica. Essa transferência de política e contratransferência pode ser uma prática eficiente, e ser inserida dentro do planejamento das políticas públicas. Isso deve constar em todo o processo das políticas públicas, corroborando com resultados finais esperados e com uma relação de confiança e aprendizado entre os diversos atores envolvidos de forma conjunta ${ }^{28}$. Além do 
custeio, o programa recebeu o fortalecimento do profissional, pois o PAS insere diretamente o profissional na rede de saúde. Destacamos o discurso conforme segue:

O custeio, o fortalecimento do vínculo do profissional da saúde na rede onde a gente pode dizer que Recife é um exemplo diferencial do restante dos país onde consequentemente nos insere diretamente de fato na rede de saúde no sistema único de saúde e isso assim acho que é o maior ganho que a gente ganha como profissional e como intervenção profissional $^{29}$.

O programa foi alvo de estudos e avaliações externas, assim como outras experiências no país similares ao PAC Recife, esses programas possuem em comum o fato de serem programas públicos, sem taxa e de base populacional, que vêm promovendo a prática de atividade física pela população. Estudos sobre esses programas proporcionaram uma maior divulgação e visibilidade municipal e nacional do PAC, conforme se justifica no relato abaixo: "Academia da Saúde influenciou a gente toda a parte documental [...] deu uma visibilidade diferenciada agora a gente não tem mais uma visibilidade só de Recife a gente tem uma visibilidade de apoio do Ministério da Saúde"23. A relação com o PAS acaba contribuindo na manutenção e ampliação do programa já existente, como demonstra podemos destacar:

E quando surgiu o Academia da Saúde valorizou, reforçou o Polo não é, que é um serviço com a unidade de saúde da família dentro de uma rede que precisa conversar com essa rede, ele precisa seguir os princípios do SUS e pensar isso com as diretrizes também do Sistema Único de Saúde e eu acho que isso reforça o Academia da Cidade que nasceu com esse desejo, mas fazia ainda de alguma forma limitada ${ }^{14}$.

O PAS busca sempre um contato com o PAC Recife por ser o modelo mais velho e pioneiro; o PAC é utilizado como nivelamento e parâmetro para discussões de procedimentos em outros programas. De acordo com Rose $^{15}$, os formuladores de políticas podem tirar lições a partir de outras políticas, que irão ajudar na resolução de seus problemas. $\mathrm{O}$ aprendizado, a partir de políticas ou programas positivos, pode gerar uma transferência com adaptações necessárias ao contexto para o qual irá ser utilizado. A partir da fala do Entrevistado 4, per- cebe-se a importância do PAC por seu pioneirismo e tempo de experiência exitosa:

A relação desses dois programas é sempre uma relação de muita troca não é o Academia da Cidade ele é o primeiro desse modelo, ele é o mais velho, ele tem experiências não é que os municípios estão passando agora e o PAC já passou e eu sempre busco esse contato com o Academia da Cidade para fazer determinados nivelamentos, discussões de procedimentos de profissionais problemas e barreiras, então tem muito esse relacionamento de troca ainda ${ }^{30}$.

Percebe-se que, mesmo após a transferência ocorrida, existe uma relação de troca e compartilhamento entre o PAS e o PAC que não foi encerrada após a implantação do PAS. É possível identificar uma contratransferência positiva para o programa. Mesmo após os indícios de transferência, percebe-se, além da contratransferência entre os programas, um ciclo de interação entre ambas as políticas, justificado principalmente pelo pioneirismo do PAC em relação ao PAS, que serve de parâmetro para programas mais novos. Essa retroalimentação advinda dos programas citados nesse estudo, que teriam utilizado o PAC como modelo e hoje retroalimenta o programa de alguma forma, fica evidente na fala do Entrevistado 3:

A parceria que existia não é com o estado, com o governo do estado, era com relação a construção dos polos, nós criamos 14 polos e demos $80 \%$ dos recursos da construção do estado e nós temos trinta e oito polos recebendo do ministério da saúde o custeio de 3 mil reais mensais por polo, não é esses são os dois, esse do ministério é um recurso mensal que nós recebemos, e o do governo do estado foi na construção do polo ${ }^{24}$.

A utilização de informações políticas já existentes pode contribuir com a formulação e gestão e diminuir as possibilidades de fracasso, pois as informações disponíveis sobre um programa existente pode explicar todo o ciclo de uma política, desde a formulação até a implantação de uma política. Nesse estudo foi evidenciado que a contratransferência é identificada posteriormente ao processo de transferência e está relacionada a uma retroalimentação que pode ser percebida a partir da identificação do recebimento de recursos para além do âmbito financeiro. 


\section{Conclusão}

Podemos concluir nesse estudo que houve uma transferência de política entre o Programa Academia da Cidade do Recife e os programas similares abordados no estudo, foi destacado que a partir do seu surgimento e consolidação, outros programas começaram a ser formulados com nomes ou moldes similares, baseados no programa do Recife, processo descrito como transferência de política que ocorre devido às contribuições que o uso de informações de políticas já existentes poderia trazer para quem pretende adotar uma política.

Percebeu-se nesse estudo uma relação de ida e volta envolvendo o PAC e o Programa Estadual Academias das Cidades do governo de Pernambuco e o Programa Federal Academia da Saúde, comprovando o fenômeno de contratransferência. A contratransferência é identificada posteriormente ao processo de transferência e está relacionada a uma retroalimentação, que pode ser percebida a partir da identificação do recebimento de recursos para além do âmbito financeiro. Tal prática representa um processo positivo dentro da política e merece destaque nos estudos sobre influências de política, pois é possível identificar benefícios para ambos os programas envolvidos. Cabe destacar também a figura dos gestores envolvidos como fator importante para ocorrência desse processo dentro da política pública, pois são ele os responsáveis pelos alinhamentos políticos e parcerias que possam contribuir para a construção, gestão e fortalecimento de uma política. Figuras importantes que deixam um legado.

\section{Conflito de interesse}

Os autores declaram não haver conflito de interesse.

\section{Contribuição dos autores}

Melo EMN e Feitosa WMN participaram da concepção inicial do estudo. Melo EMN, participou da concepção inicial do estudo, redação e foi responsável pela busca da literatura, coleta de dados e conclusão e escrita do estudo. Feitosa WMN, Guarda FRB e Santos FAS participaram da revisão crítica do texto.

\section{Referências}

1. Barros MVG, Guarda FRB, Feitosa WMN, Lemos EC, Silva CRM. Programs and interventions for physical activity promotion in the Brazilian Unified Health System: a research object that starts to be unveiled. Rev Bras Ativ Fís Saúde. 2016;21(5):385-7.

2. Brasil. Ministério da Saúde. Secretaria de Vigilância em Saúde. Secretaria de Atenção à Saúde. Política Nacional de Promoção da Saúde. 3a ed. Brasília: Ministério da Saúde, 2010.
3. Hallal PC. Physical activity and health in Brazil: research, surveillance and policies. Cad Saúde Pública. 2014;30:2487-89.

4. Heath GW, Parra DC, Sarmiento OL, Andersen LC, Owen N, Goenka S, et al. Evidence-based intervention in physical activity: lessons from around the world. The Lancet. 2012;380(9838):272-81.

5. Malta DC, Neto OLM, Rocha D, Castro AM, Reis AAC, Akerman M. Política Nacional de Promoção da Saúde (PNPS): capítulos de uma caminhada ainda em construção. Cien Saúde Colet. 2016;21(6):1683-94.

6. Prefeitura Municipal do Recife. Decreto $n^{\circ} .19 .808$, de 03 de abril de 2003. Institui e regulamenta o Programa Academia da Cidade. Legiscidade Recife, 2003.

7. Cruz DA, Albuquerque G, Damascena W. Programa Academia da Cidade do Recife: minimizando contrastes sociais. DDS Brasil; 2013. [citado em 2013 fev. 6]. Disponível em: http://dssbr.org/site/experiencias/programa-academiada-cidade-do-recife-minimizando-contrastes-sociais/.

8. Feitosa W. Programas de promoção da atividade física: estudo de caso acerca da implementação do Programa Academia da Cidade (PAC) em Recife entre 2002 e 2014 [tese de doutorado em Saúde Pública]. Recife: Fundação Oswaldo Cruz; 2015.

9. Dolowitz D, Marsh D. Who learns what from whom: a review of the policy transfer literature. Polit Stud. 1996;44(2):343-57.

10. Dolowitz D, Marsh D. Learning from Abroad: The Role of Policy Transfer in Contemporary Policy-Making. Governance. 2000;13(1):5-23.

11. Evans M. Policy transfer in critical perspective. Policy Stud J. 2009;30(3):243-68.

12. Minayo MCS. O desafio do conhecimento: pesquisa qualitativa em saúde. 9a ed. São Paulo: Hucitec, 2006.

13. Kvale S. Interviews: an introduction to qualitative research interviewing. Thousand Oaks: Sage, 1996.

14. Entrevistado 1. Entrevista I. [Entrevista cedida a] Érica Nascimento. Recife, ago. 2018.1 arquivo, mp3, 46m54s.

15. Rose R. What is lesson-drawing? Int J Public Pol. 1991;11(1):3-30.

16. Stone D. Transfer agents and global networks in the "transnationalization" of policy. J Eur Public Policy. 2004;11(3):545-68.

17. Hallal PC, Carvalho YM, Tassitano RM, Tenório MCM, Warschauer M, Reis RS, et al. Avaliação quali-quantitativa do programa Academia da Cidade, Recife (PE): concepções dos professores. Rev Bras Ativ Fís Saúde. 2009;14(1):9-14.

18. Knuth AG, Simões EJ, Reis RS, Hallal PC, Cruz DKA, Zanchetta LM, et al. Avaliação de programas de atividade física no Brasil: uma revisão de evidências em experiências selecionadas. Secretaria de Vigilância em Saúde/MS. n. 4, 2010. Disponível em: https://edisciplinas. usp.br/pluginfile.php/372224/mod_resource/content/1/ Avalia\%C3\%A7\%C3\%A3oMSBrasilProgramasdeAF.pdf

19. Simoes EJ, Hallal P, Pratt M, Ramos L, Munk M, Damascena W, et al. Effects of a community-based, professionally supervised intervention on physical activity levels among residents of Recife, Brazil. Am J Public Health. 2009;99(1):68-75.

20. Ghesti JP, Silva SPA. Abordagem territorial do Programa Leader na Europa e sua transferência institucional ao contexto latino-americano: uma análise crítica. Boletim Regional, Urbano e Ambiental. 2016;14:219-31. 
21. Fontoura Y, Guedes AL. Governança global e transferência de política: do protocolo de Cartagena à política nacional de biossegurança. Revista de Administração Pública. 2013;47(1):3-23.

22. Pernambuco. Decreto no 31.140, de 6 de dezembro de 2007. Institui o Programa Academia das Cidades no âmbito do Poder Executivo Estadual e dá outras providências. Diário Oficial de Pernambuco, Recife, PE, 7 dez. 2007. No 230, p. 3.

23. Entrevistado 2. Entrevista II. [Entrevista cedida a] Érica Nascimento. Recife, ago. 2018. 1 arquivo, mp3, 58m42s.

24. Entrevistado 3. Entrevista III. [Entrevista cedida a] Érica Nascimento. Recife, set. 2018.1 arquivo, mp3, 17m42s.

25. Brasil. Ministério da Saúde. Portaria no 1.707, de 23 de setembro de 2016. Redefine as regras e os critérios referentes aos incentivos financeiros de investimento para construção de polos; unifica o repasse do incentivo financeiro de custeio por meio do Piso Variável da Atenção Básica (PAB Variável); e redefine os critérios de similaridade entre Programas em desenvolvimento no Distrito Federal e nos Municípios e o Programa Academia da Saúde. Diário Oficial da União, Brasília, 26 nov. 2016.
26. Brasil. Ministério da Saúde. Portaria no 2.681, de 7 de novembro de 2013. Redefine o Programa Academia da Saúde no âmbito do Sistema Único de Saúde (SUS). Diário Oficial da União, 7 nov. 2013.

27. Sá GBAR, Dornelles GC, Cruz KG, Amorim RCA, Andrade SSCA, Oliveira TP, et al. O Programa Academia da Saúde como estratégia de promoção da saúde e modos de vida saudáveis: cenário nacional de implementação. Ciênc Saúde Colet. 2016;21(6):1849-60.

28. Oliveira JAP. Challenges of public policy planning: different views and practices. Revista de Administração Pública. 2006;40(2):273-87.

29. Entrevistado 5. Entrevista V. [Entrevista cedida a] Érica Nascimento. Recife, out. 2018.1 arquivo, mp3, 15m17s.

30. Entrevistado 4. Entrevista IV. [Entrevista cedida a] Érica Nascimento. Recife, ago. 2018. 1 arquivo, mp3, 9m27s.

Recebido: 30/07/2020

Aprovado: 07/12/2020

\section{Como citar este artigo:}

Melo EMN, Guarda FRB, Santos FAS, Feitosa WMN. Programa Academia da Cidade do Recife: análise da contratransferência de política. Rev Bras Ativ Fis Saúde. 2020;25:e0174. DOI: 10.12820/rbafs.25e0174 